Research Article

\title{
Biochemical and molecular characterization of 3-Methylcrotonylglycinuria in an Italian asymptomatic girl
}

\author{
Carla Cozzolino $^{1 \S}$, Guglielmo RD Villani ${ }^{1,2, \S}$ (D), Giulia Frisso ${ }^{1,2}$, Emanuela Scolamiero ${ }^{1}$, Lucia Albano ${ }^{1}$, \\ Giovanna Gallo ${ }^{1}$, Roberta Romanelli ${ }^{1}$ and Margherita Ruoppolo ${ }^{1,2}$ \\ ${ }^{1}$ CEINGE Biotecnologie Avanzate, Naples, Italy. \\ ${ }^{2}$ Dipartimento di Medicina Molecolare e Biotecnologie Mediche, Università degli Studi di Napoli, "Federico \\ II", Naples, Italy.
}

\begin{abstract}
3-Methylcrotonylglycinuria is an organic aciduria resulting from deficiency of 3-methylcrotonyl-CoA carboxylase (3-MCC), a biotin-dependent mitochondrial enzym carboxylating 3-methylcrotonyl-CoA to 3-methylglutaconyl-CoA during leucine catabolism. Its deficiency, due to mutations on MCCC1 and MCCC2 genes, leads to accumulation of 3-methylcrotonyl-CoA metabolites in blood and/or urine, primarily 3-hydroxyisovaleryl-carnitine $(\mathrm{C} 5-\mathrm{OH})$ in plasma and 3-methylcrotonyl-glycine (3-MCG) and 3-hydroxyisovaleric acid (3-HIVA) in the urine. The phenotype of 3-MCC deficiency is highly variable, ranging from severe neurological abnormalities and death in infancy to asymptomatic adults. Here we report the biochemical and molecular characterization of an Italian asymptomatic girl, positive for the newborn screening test. Molecular analysis showed two mutations in the MCCC2 gene, an already described missense mutation, c.691A > T (p.I231F), and a novel splicing mutation, c.1150-1G > A. We characterized the expression profile of the splice mutation by functional studies.
\end{abstract}

Keywords: 3-Methylcrotonylglycinuria, MCCC2 mutations, 3-methylcrotonyl-CoA carboxylase deficiency, newborn screening, organic aciduria.

Received: April 05, 2017; Accepted: October 22, 2017.

\section{Introduction}

3-Methylcrotonylglycinuria (OMIM \#210200, \#210210) is an organic aciduria resulting from deficiency of 3-methylcrotonyl-CoA carboxylase (3-MCC, EC 6.4.1.4), a biotin-dependent mitochondrial enzyme involved in the fourth step of the catabolic pathway of the amino acid leucine (Sweetman and Williams, 2001) (Figure 1). Its deficiency leads to accumulation in blood and/or urine of 3-methylcrotonyl-CoA metabolites, primarily 3-hydroxyisovaleryl-carnitine $(\mathrm{C} 5-\mathrm{OH})$ in plasma and 3methylcrotonyl-glycine (3-MCG) and 3-hydroxyisovaleric acid (3-HIVA) in urine (Arnold et al., 2012); patients often show a secondary carnitine deficiency due to its conjugation to 3-HIVA. The 3-methylcrotonylglycinuria is the most frequent organic aciduria detected by an increase of $\mathrm{C} 5-\mathrm{OH}$ in tandem mass spectrometry-based newborn screening programs (NBS) (Gallardo et al., 2001). Its prevalence is highly variable ranging from 1:2,400 in the Faroe Islands (Thomsen et al., 2015) to 1:68,333 in the Chinese

Send correspondence to Guglielmo RD Villan. Dipartimento di Medicina Molecolare e Biotecnologie Mediche, Università degli Studi di Napoli, "Federico II", Via S. Pansini 5, 80131 Naples, Italy. E-mail: guglielmorosariodomeni.villani@unina.it.

${ }^{\S}$ These authors equally contributed to the work population (Yang et al., 2015). Interestingly, the prevalence could be lower in Brazil, and in fact, studies on frequencies of primary disorders of organic acid metabolism in high-risk Brazilian patients evidenced a frequency of only $0.92 \%$ of 3 -Methyl-crotonylglycine-CoA carboxylase deficiency (two patients among 218 with organic aciduria) (Wajner et al., 2002; Wajner et al., 2009).

3-MCC deficiency is inherited as an autosomal recessive trait due to mutations affecting one of the two genes, $M C C C 1$ and $M C C C 2$, encoding for two subunits, MCCa and $\mathrm{MCC} \beta$, respectively, that form the 3-MCC protein (Baumgartner et al., 2001). The $\alpha$ subunit includes the biotin carboxylase (BC) domain and the biotin carboxyl carrier protein domain covalently bound with a biotin prosthetic group, while the $\beta$ subunit contains the carboxyltransferase (CT) domain (Grünert et al., 2012).

The clinical phenotype of 3-MCC deficiency is highly variable, ranging from severe neurological abnormalities and death in infancy (Baykal et al., 2005) to asymptomatic adults (Dantas et al., 2005; Forsyth et al., 2016). A severe presentation of 3-MCC deficit may include the Reye-like illness, ketoacidosis, hypoglycemia, hyperammonemia, psychomotor retardation, seizures, symptoms of cardiorespiratory failure and coma. Mild phenotypes of- 


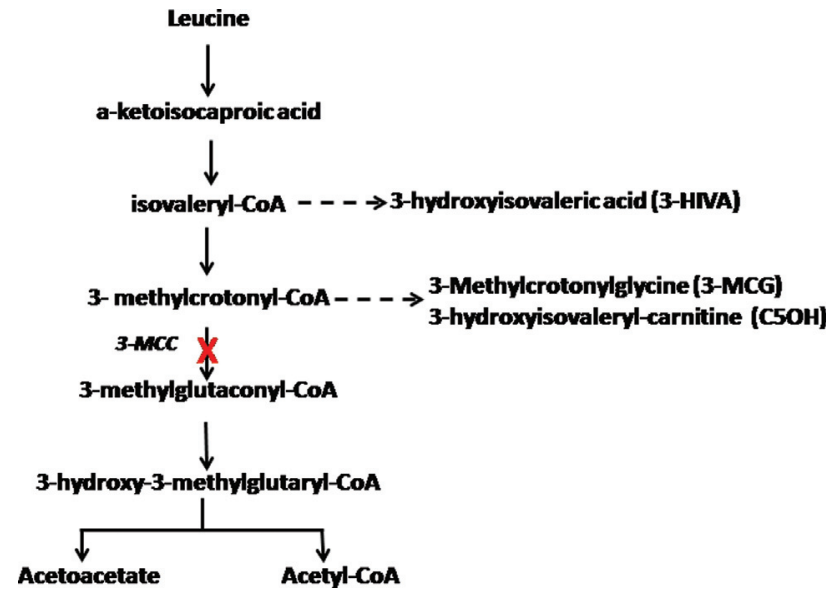

Figure 1 - The 3-MCC-catalyzed reaction and its position in the leucine catabolic pathway. The dashed arrow indicates the metabolites that accumulate due to deficiency of 3-MCC.

ten include fatigue and weakness during catabolic episodes or mild developmental delay. Moreover, cardiomyopathy, brain atrophy and fatty infiltration of liver or muscle may also occur (Grünert et al., 2012). Nonetheless, most of the patients diagnosed by NBS as affected by 3-MCC deficiency appear to be asymptomatic during the whole life (Forsyth et al., 2016).

In this report we present the biochemical and molecular characterization of an Italian girl, positive for the newborn screening test. Molecular analysis showed two mutations in $M C C C 2$ gene, a missense mutation and a splicing mutation. We characterized the expression profile of the novel splice mutation by functional studies.

\section{Materials and Methods}

\section{Biochemical analysis}

The biochemical characterization of the patient was obtained by analyzing amino acids and acylcarnitines in dried blood spot (DBS) (at three days from birth) and in serum (at seven days from birth), organic acids in urine and biotinidase enzyme activity on dried blood spot. For these analyses, labeled standards of amino acids and acylcarnitines were purchased from Cambridge Isotope Laboratories (Andover, MA, USA). External standard blood spots from acylcarnitines and amino acids were from CDC (Atlanta, GA, USA).

Amino acids and acylcarnitines were analyzed as butyl esters by using a triple quadrupole tandem mass spectrometer API 4000 (AB/Sciex) connected to an Agilent 1200 Series autosampler as described (Scolamiero et al., 2014; Scolamiero et al., 2015). Briefly, DBS from the newborn was punched into a $1.5 \mathrm{~mL}$ test tube and $200 \mu \mathrm{L}$ of methanol containing labeled standards were added.

The standard concentrations were in the 500-2500 $\mu \mathrm{mol} \mathrm{L}{ }^{-1}$ range for amino acids, and in the $7.6-152 \mu \mathrm{mol} \mathrm{L}^{-1}$ range for acylcarnitines. After $20 \mathrm{~min}$ of shaking at room temperature, the samples were dried under a nitrogen flow. The extracted acylcarnitines and amino acids were derivatized to butyl esters with $80 \mu \mathrm{L}$ of $3 \mathrm{~N} \mathrm{HCl}$ in $\mathrm{N}$ butanol at $65{ }^{\circ} \mathrm{C}$ for $25 \mathrm{~min}$. After derivatization, the samples were dried under nitrogen flow and resuspended in 300 $\mu \mathrm{L}$ of acetonitrile/water (70:30) containing $0.1 \%$ formic acid. Forty microliters were injected in the flow injection analysis mode for the MS/MS analysis. For serum analysis, $10 \mu \mathrm{L}$ of the patient's serum was spotted on a Schleicher \&Schuell 903 grade filter paper (Whatman, Dassel, Germany) and dried overnight at room temperature. The next day, the filter paper was punched into a $1.5 \mathrm{~mL}$ test tube and processed as described for DBS. Each sample was analyzed on an API 4000 triple quadrupole mass spectrometer (Applied Biosystems-Sciex, Toronto, Canada) coupled with the high performance liquid chromatograph Agilent 1100 series (Agilent Technologies, Waldbronn, Germany), as described (Scolamiero et al., 2015).

The extraction and quantification of the urinary organic acids was performed as previously reported (Villani et al., 2016). Urine samples were quantified for creatinine in order to determine the amount of sample to be analyzed, equivalent to $0.5 \mu \mathrm{mol}$ of creatinine, and $\mathrm{NaOH} 30 \%$ was added to the samples up to a final $\mathrm{pH}$ of 14.0. Subsequently, $500 \mu \mathrm{L}$ of hydroxylamine hydrochloride $2.5 \mathrm{~g} / \mathrm{L}$ (in water) were added to each tube and samples were allowed to react at $60{ }^{\circ} \mathrm{C}$ for $1 \mathrm{~h}$. After acidification with $\mathrm{H}_{2} \mathrm{SO}_{4} 2.5 \mathrm{~N}$ to a final $\mathrm{pH}$ of 1.0 internal standards were added: 10,20 or $20 \mu \mathrm{l}$ of a $100 \mu \mathrm{g} / \mathrm{mL}$ solution of dimethylmalonic acid, tropic acid and pentadecanoic acid (PDA), respectively. Three extractions of the organic acids were performed by mixing vigorously each sample with $2 \mathrm{~mL}$ of ethyl acetate; the organic phases were transferred to another tube, and approximately $1 \mathrm{~g}$ of $\mathrm{Na}_{2} \mathrm{SO}_{4}$ was added. After 1 hour on bench, samples were centrifuged and the organic phase was transferred to a new clean glass tube to be completely evaporated under a gentle nitrogen flow. Fifty microliters of BSTFA was added to each dried sample and the derivatization reaction was performed at $60{ }^{\circ} \mathrm{C}$ for $30 \mathrm{~min}$. Finally, $1 \mu \mathrm{L}$ of the sample was used for GC/MS injection. Analyses were performed on an Agilent Technologies Model 7890A gas chromatograph combined with a 5975C mass spectrometer system and equipped with a split-mode capillary injection port held at $280{ }^{\circ} \mathrm{C}$ with a split ratio of 10:1. The column (Agilent J\&W GC column HP-5MS) was directly interfaced to the ion source. The oven temperature was programmed from $70{ }^{\circ} \mathrm{C}$ to $280{ }^{\circ} \mathrm{C}$ at a rate of 10 ${ }^{\circ} \mathrm{C} / \mathrm{min}$, and the helium flow program was $1 \mathrm{~mL} / \mathrm{min}$. Data were acquired by repetitive scanning over a range of 50-550 amu. The retention time and area of each peak was automatically determined and printed out by the MSD Productivity Chemstation software (Agilent Technologies). Quantitation of each analyte was performed referring to the injected PDA quantity according to Tanaka et al. (1980a, b). The concentration of organic acids was normalized to 
the creatinine concentration of the urine sample and expressed as mmol organic acid/mol creatinine.

Neonatal screening for biotinidase deficiency was performed by colorimetric semi-quantitative analysis (Heard et al., 1984).

\section{Genetic analysis}

Genomic DNA was extracted from peripheral blood leukocytes of the patient and her parents using the "Nucleon" procedure (GE Healthcare, Little Chalfont, UK). Molecular analysis of the MCCC1 (methylcrotonoyl-CoA carboxylase 1, alpha) and MCCC2 (methylcrotonoyl-CoA carboxylase 2, beta) genes was performed by PCR, followed by direct sequencing of all exons and exon-intron boundaries, including 5'- and 3'-UTR regions (primers, sequences and condition for PCR amplification are available on request). PCR products were examined for sequence variations using a Big Dye Primer Cycle Sequencing kit and an ABI 3730 DNA Analyzer (Applied Biosystems). Nucleotide positions were numbered on the basis of the cDNA sequence (GenBank, MCCC1: NM_020166.4 and MCCC2: NM_022132.4) according to the nomenclature of den Dunnen and Antonarakis (2001).

Since the patient's RNA was not available for studying the effect of the c.1150-1G $>$ A novel variation on splicing pattern, we used an artificial minigene construct designated pMGene (Amato et al., 2012) kindly donated by F. Amato. A DNA fragment of approximately $1 \mathrm{~kb}$, including the c.1150-1G > A variation in the $M C C C 2$ gene, was amplified from the genomic DNA of the patient and cloned into the pMGene: the PCR product was obtained using the Expand High Fidelity PCR System (Roche Life Science, Penzberg, Germany), then digested by the KpnI restriction enzyme and cloned into the KpnI-digested dephosphorylated pMGene vector (primers, sequences and conditions for PCR amplification and cloning are available on request). All clones were sequenced, and a wild-type and a mutated form were retained for expression experiments.

tsA201 cells were grown in DMEM supplemented with $10 \%$ fetal bovine serum and $1 \%$ penicillin/streptomycin in a humidified, $5 \% \mathrm{CO}_{2}$ atmosphere at $37^{\circ} \mathrm{C}$. They were transfected with $2 \mu \mathrm{g}$ of WT-pMGene or $2 \mu \mathrm{g}$ of MUT-pMGene plasmids, using FuGENE 6 (Roche), according to the manufacturer's instructions. Forty-eight hours after transfection, cells were collected and total RNA was extracted using TriPure Isolation Reagent (Roche Applied Science). Next, $1 \mu \mathrm{g}$ of total RNA was reverse transcribed using SuperScript VILO cDNA synthesis kit (Life Technologies). Finally, 200 ng of cDNA were amplified using the forward primer pMG-GFP-FW (5'-ACGACGGCAACTACAAGACC-3') and reverse primer pMG-bglob-ex3-REV (5'-CACACCAGCCACC ACTTTC-3') in a previously described protocol (Catanzano et al., 2010). The PCR products were subjected to bidirectional cycle sequencing using a Big Dye Primer Cycle Sequencing kit and an ABI 3730 DNA Analyzer (Applied Biosystems).

To verify the potential role of the variants identified by genetic screening, we also checked their frequency in population genomes databases, such as 1.000 Genomes (http://www.internationalgenome.org/) and ExAC (http://exac.broadinstitute.org/) consortia, which aggregate and harmonize exome sequencing data from a variety of large-scale sequencing projects. In silico bio-informatic evaluations were performed using Alamut Focus version 0.9 , a licensed software package available from Interactive Biosoftware (www.interactive-biosoftware.com). Genomic sequences (WT and mutant) were processed by this predictor software using five splicing (SpliceSiteFinderlike, MaxEntScan, Neural Network Splice, GeneSplicer, and Human SplicingFinder) and three missense prediction algorithms (SIFT, Polyphen-2, MutationTaster). The robustness of these bio-informatic tools is widely accepted (Frisso et al., 2016).

\section{Results}

The patient was a girl born to non-consanguineous parents after a full term normal delivery of dizygotic twins, weighing $\backslash 2.57 \mathrm{~kg}$. Physical examination was normal and she was discharged from hospital 72 hours after birth. However, while the amino acid profile was normal (data not shown), the acylcarnitine profile, obtained from dried blood spot during the newborn screening, presented a significant increase in 3-hydroxyisovaleryl-carnitine (C5$\mathrm{OH})$, along with a normal free carnitine $(\mathrm{C} 0)$ concentration (Table 1). Similarly, a high concentration of $\mathrm{C} 5-\mathrm{OH}$ with normal $\mathrm{C} 0$ concentration was reported as a consequence of liver immaturity in a false-positive case, having a blood C5-OH value normalized at five months of life (RangelCórdova et al., 2009). In addition, a recent study pointed

Table 1 - Biomarkers found altered in the analyzed patient. These were quantified by LCMSMS analysis on dried blood spot and serum, by GCMS analysis in urine

\begin{tabular}{lccc}
\hline & Dried Blood Spot $(\mathrm{DBS})(\mu \mathrm{mol} / \mathrm{L})$ & Serum $(\mu \mathrm{moli} / \mathrm{L})$ & Urine $(\mathrm{mmol} / \mathrm{mol} \mathrm{crea})$ \\
\hline $\mathrm{C} 5 \mathrm{OH}$ & $4.09(<0.4)$ & $4.58(<0.13)$ & - \\
$\mathrm{C} 0$ & $15.29(11-51)$ & $8.7(10-45)$ & - \\
3-hydroxy-isovaleric acid & - & - & $70(0-1.3)$ \\
3-methylcrotonylglycine & - & - & 441 (n.d.) \\
\hline
\end{tabular}

reference intervals are reported in brackets n.d.: not detectable. 
out that the $\mathrm{C} 5-\mathrm{OH}$ level found in newborn screening is not sufficient for diagnostic or predictive purposes (Forsyth et al., 2016). However, the detection of a $\mathrm{C} 5-\mathrm{OH}$ concentration well above our cut-off led us to deepen the clinical evaluation and to start prompt confirmatory testing in order to investigate whether an inborn error of metabolism was associated to the biochemical alteration. The increase of C5-OH acylcarnitine in blood/plasma may be ascribed to different pathologies, since several enzymes (3-methylcrotonyl-CoA carboxylase, 3-methylglutaconic hydratase, 3-hydroxy-3-methylglutaryl-CoA lyase, 2-methyl-3hydroxybutyril-CoA dehydrogenase, beta-ketothiolase, holocarboxylase synthetase and biotinidase) may be defective either in the leucine or the isoleucine catabolic pathway (Sweetman and Williams, 2001).

Although the child remained asymptomatic, the acylcarnitine analysis performed on serum at 7 days from birth confirmed the increase of $\mathrm{C} 5-\mathrm{OH}$, but showed also a significant decrease of $\mathrm{C} 0$ (Table 1). As stated above, elevation of $\mathrm{C} 5-\mathrm{OH}$ acylcarnitine levels in blood spot is not unique to 3-MCC deficiency. Conditions such as 3-hydroxy-3- methylglutaryl-coenzyme A lyase deficiency, $\beta$-ketothiolase deficiency, multiple carboxylase deficiency resulting from holocarboxylase synthetase or biotinidase deficiency, 2-methyl 3-hydroxybutyric acidemia, and 3-methylglutaconic aciduria may occur with elevation of $\mathrm{C} 5 \mathrm{OH}$ acylcarnitine in asymptomatic infants. Therefore, the differential diagnosis between different organic acidurias must be considered in the presence of isolated elevation of $\mathrm{C} 5 \mathrm{OH}$ acylcarnitine levels. In our case, the analysis of the newborn urinary organic acids showed an abnormal profile characterized by an increase of 3-hydroxyisovaleric acid and a massive excretion of 3-methyl-crotonylglycine (Table 1, Figure 2). A biotinidase enzyme assay on dried blood spot showed normal enzyme activity. A possible maternal origin of the metabolic alteration, due to transplacental transfer of C5OH (Rangel-Córdova et al., 2009), was investigated and excluded by the analysis of the mother's serum acylcarnitine and urine organic acids (data not shown). These results were consistent with the diagnosis of 3methyl-crotonyl-CoA carboxylase deficiency and justified the molecular diagnosis.

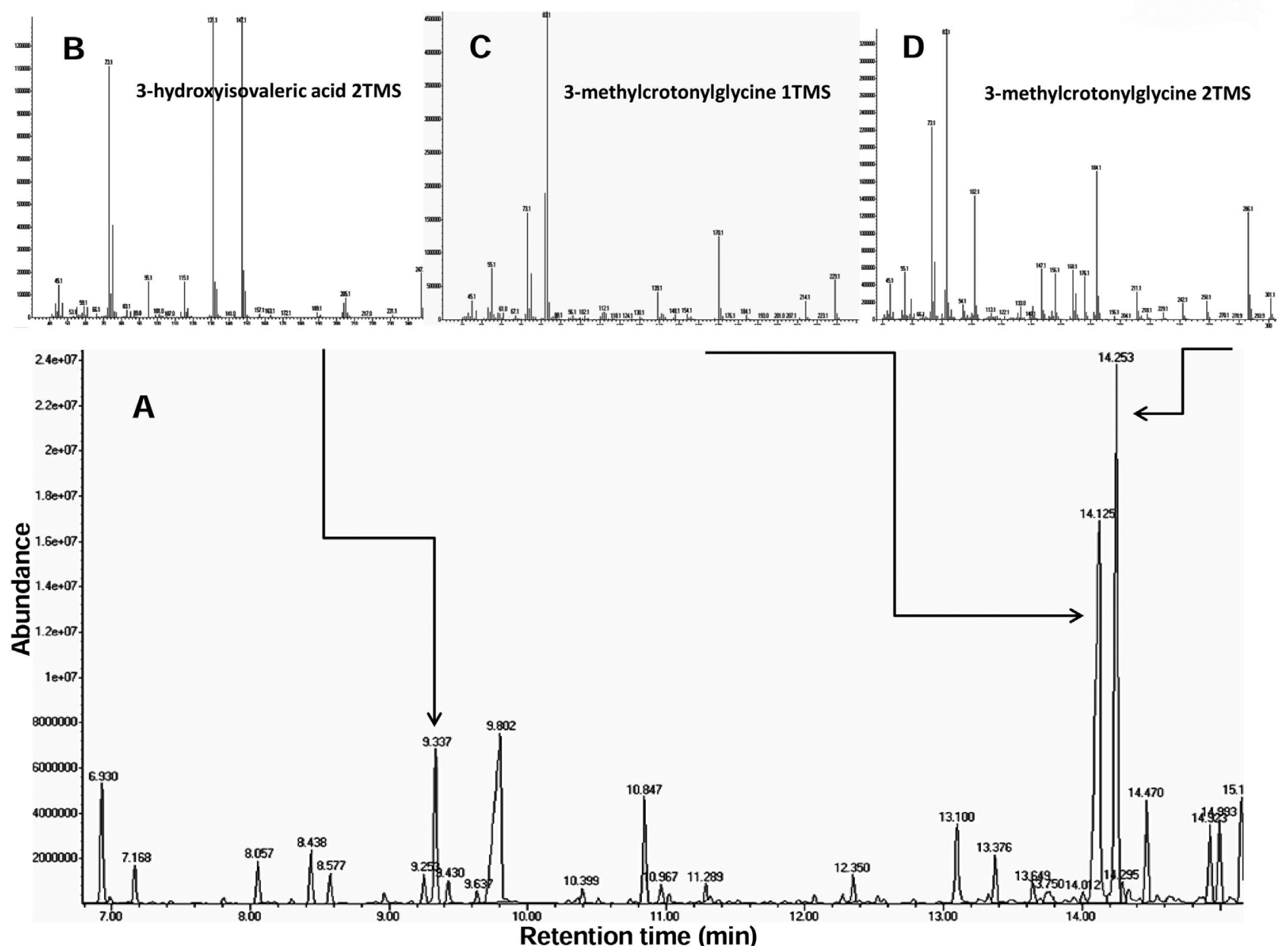

Figure 2 - Patient's urinary organic acids profile. (A) Partial organic acids profile from urine; arrows point to the main altered metabolites; (B-D) mass spectra for these organic acids. 1TMS and 2TMS refer to the presence of 1 or 2 trimethylsylil ester groups, respectively, added during the derivatization reaction. 
3-MCC deficiency is due to mutations in either the $M C C C 1$ or $M C C C 2$ gene. The Human Gene Mutation Database (HMGD) (Stenson et al., 2014) currently includes 103 MCCC1 mutations and 113 MCCC2 mutations, most of which are missense mutations. Several of these mutations were recently reported in the Portuguese newborn screening program (Fonseca et al., 2016). In our patient, the sequencing of the coding regions of $M C C C 1$ and $M C C C 2$ genes showed that she was a compound heterozygote for two mutations in the $M C C C 2$ gene: a missense mutation in exon 7, namely c.691A > T (p.I231F), and a possible splicing mutation in intron $12, \mathrm{c} .1150-1 \mathrm{G}>\mathrm{A}$ transition, inherited from the patient's mother and father, respectively. Her twin brother did not carry these mutations (Figure 3). The c.691A > T (p.I231F) mutation was annotated in the HGMD database (http://www.hgmd.cf.ac.uk/), and showed an MAF $=0.02 \%$ and $0.0008 \%$ in the 1000 Genomes and ExAC databases, respectively. The c.1150-1G > A variant was absent from all the examined databases (HGMD, 1000 Genomes and ExAC).

The c.691A > T mutation causes the replacement of isoleucine 231 , a non-polar amino acid, with phenylalanine, an aromatic amino acid (p.I231F). This variation has been described for the first time, in homozygosis, in an asymptomatic Iranian patient (Al-Jasmi et al., 2016), positive in newborn screening (high levels of $\mathrm{C} 5-\mathrm{OH}$ ). $\mathrm{I} 231$ is a highly conserved amino acid, and a deleterious effect is predicted for $1231 \mathrm{~F}$-mutated protein by in silico analysis performed using Alamut software (Supplemental Table $\mathrm{S} 1)$.

I

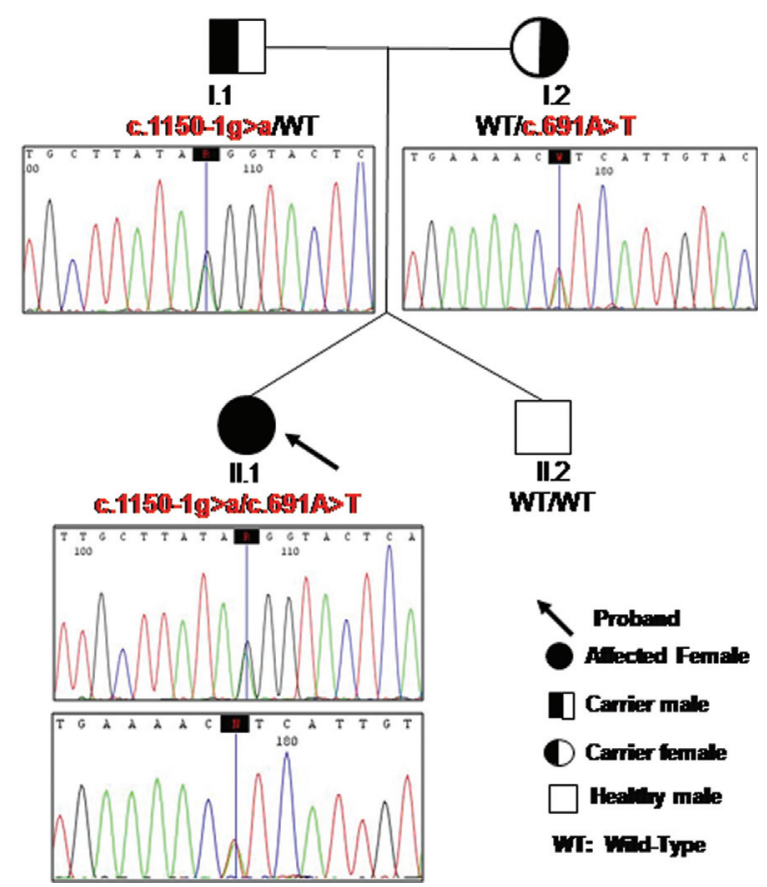

Figure 3 - Pedigree of the patient with MCC deficiency. Sequence electropherograms of exon 7 and intron 12/exon 13 boundary of the $M C C C 2$ gene are shown for the patient and her parents.
The novel variant c. $1150-1 \mathrm{G}>\mathrm{A}$ is located in the acceptor splice site of intron 12 of the $M C C C 2$ gene and presumably resulted in the skipping of exon 13 , as predicted by the bioinformatics program (Table S1). To verify this prediction, we used the minigene system, since the patient's RNA from leucocytes for transcript analysis was unavailable. The RT-PCR and sequence analysis of mRNA, extracted from cell lines transfected with WT or mutated minigene constructs, revealed the presence of two transcripts (normal and alternative) in the pMGene-MCCC2c.1150-1G $>A$, and only one (normal) in the pMGeneMCCC2-WT (Figure 4). The alternative transcript showed the skipping of exon 13, resulting in a frame shift starting at codon Gly384, and a stop codon 32 positions downstream (p.G384Dfs*32). The presence of these two mutations in the gene $M C C C 2$ definitively confirmed the diagnosis of 3-MCC deficiency for the patient.

\section{Discussion}

Deficiency of 3-methylcrotonyl-CoA carboxylase does not show a good genotype-phenotype correlation, since deleterious mutations in homozygosis do not cause a clinical phenotype, and even complete absence of 3-MCC activity leads to clinical manifestations in a rather small subgroup of individuals (Dantas et al., 2005; Morscher et al., 2012).

In our study, an asymptomatic girl showed a significant alteration in biochemical markers. A persistent increase of $\mathrm{C} 5-\mathrm{OH}$ levels was detected by DBS and serum analysis, and a small decrease of C0 levels was demonstrated by serum acylcarnitine analysis at seven days from birth. An increase in 3-hydroxyisovaleric acid and a massive excretion of 3-methyl-crotonylglicine resulted from urinary organic acid quantization. The decrease, in serum, of $\mathrm{C} 0$ levels after four days from the first determination on DBS appears to be consistent with the persistent high levels of 3OH-isovaleryl-carnitine $(\mathrm{C} 5-\mathrm{OH})$, which may gradually deplete the reserves of free carnitine.

Molecular analysis confirmed the diagnosis of 3MCC deficiency. Interestingly, the patient presented two $M C C C 2$ mutations: the splicing mutation generating a frameshift most likely produces a null allele; the missense mutation (p.I231F) is located in the CT domain. It is difficult to establish the pathogenic role of a missense mutation; however, the deleterious effect predicted for the I231Fmutated protein by in silico analysis suggests that it may impair the carboxyltransferase activity.

Although the clinical relevance of the biochemical alterations related to this pathology is yet under evaluation, it is unquestionable that, in some patients, the 3 -MCC defect results in severe clinical manifestations, mainly neurological impairment and cardiorespiratory failure, which can lead to death in infancy (Bannwart et al., 1992; Dantas et al., 2005), or acquires relevance later in the patient's life. In these cases it is hypothesized that the 3-MCC enzyme de- 
A

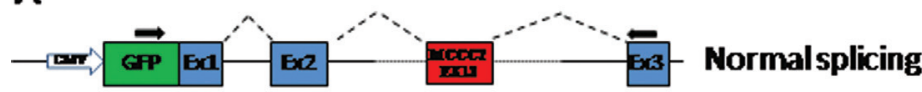

$\rightarrow$ GFP
B

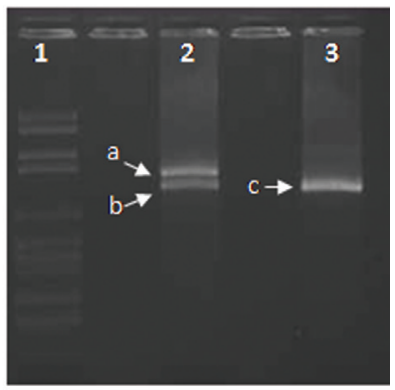

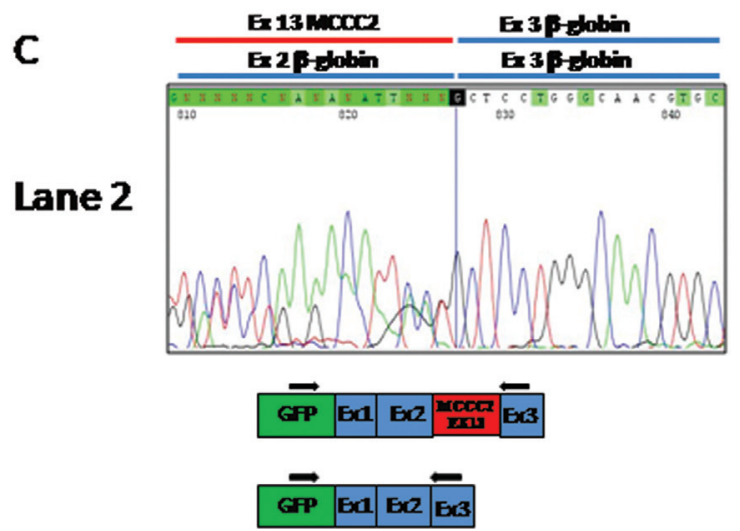

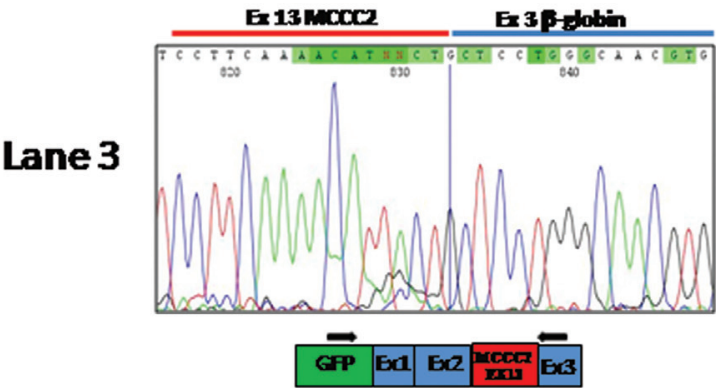

Figure 4 - Minigene construct and RT-PCR results obtained in expression studies. (A) The minigene construct (pMGene) used in the study: a DNA fragment of approximately $1 \mathrm{~kb}$ was directly amplified from the genomic DNA of the patient and cloned into the pMGene as decribed in the Results section. All clones were sequenced, and a wild-type and a mutated clone were used for expression experiments. (B and C) The splicing pattern, evaluated by RT-PCR and sequence analysis of mRNA extracted from cell lines transfected with WT or mutated minigene constructs. Lane 1: molecular weight marker, lane 2: RT-PCR of RNA obtained using the mutant clone; lane 3: RT-PCR of RNA obtained from the normal clone.

fect cooperates with environmental factors or modifying genes to establish the pathological evolution of the disease (Baumgartner, 2005). Moreover, recent studies suggest that alterations of the cellular redox homeostasis may potentially be involved in the pathophysiology of 3-MCC deficiency (Zanatta et al., 2013). Therefore, the accumulation of oxidative damage during the patient's life could contribute to explain neurological symptoms in late onset 3-MCC forms, with or without previous crises of metabolic decompensation.

With this in mind, aim of the present report is, first of all, to emphasize the importance of differential diagnosis among much more severe metabolic diseases, in asymptomatic infants with high levels of $\mathrm{C} 5 \mathrm{OH}$. Moreover, a constant monitoring is recommended for patients presenting with 3-methylcrotonyl-CoA carboxylase deficiency, particularly asymptomatic newborns. The possible absence of symptoms should not lead parents and paediatrics to underestimate the pathology. It is also suggested, despite the apparent absence of clinical manifestations, to evaluate the opportunity of starting a preventive therapy in patients identified by abnormal biochemical findings, in order to reduce the risk of neurological delay and cardiomyopathy.

\section{References}

Al-Jasmi FA, Al-Shamsi A, Hertecant JL, Al-Hamad SM and Souid AK (2016) Inborn errors of metabolism in the United Arab Emirates: Disorders detected by newborn screening (2011-2014). JIMD Rep 28:127-135.

Amato F, Bellia C, Cardillo G, Castaldo G, Ciaccio M, Elce A, Lembo F and Tomaiuolo R (2012) Extensive molecular analysis of patients bearing CFTR-related disorders. J Mol Diagn 14:81-9.

Arnold GL, Salazar D, Neidich JA, Suwannarat P, Graham BH, Lichter-Konecki U, Bosch AM, Cusmano-Ozog K, Enns G, Wright EL et al. (2012) Outcome of infants diagnosed with 3-methyl-crotonyl-CoA-carboxylase deficiency by newborn screening. Mol Genet Metab 106:439-441.

Bannwart C, Wermuth B, Baumgartner R, Suormala T and Weismann UN (1992) Isolated biotin-resistant deficiency of 3methylcrotonyl-CoA carboxylase presenting as a clinically severe form in a newborn with fatal outcome. J Inherit Metab Dis 15:863-868.

Baumgartner MR, Almashanu S, Suormala T, Obie C, Cole RN, Packman S, Baumgartner ER and Valle D (2001) The molecular basis of human 3-methylcrotonyl-CoA carboxylase deficiency. J Clin Invest 107:495-504.

Baykal T, Gokcay GH, Ince Z, Dantas MF, Fowler B, Baumgartner MR, Demir F, Can G and Demirkol M (2005) Consanguineous 3-methylcrotonyl-CoA carboxylase deficiency: Early-onset necrotizing encephalopathy with lethal outcome. J Inherit Metab Dis 28:229-233. 
Catanzano F, Ombrone D, Di Stefano C, Rossi A, Nosari N, Scolamiero E, Tandurella I, Frisso G, Parenti G, Ruoppolo $\mathrm{M}$ et al. (2010) The first case of mitochondrial acetoacetyl-CoA thiolase deficiency identified by expanded newborn metabolic screening in Italy: The importance of an integrated diagnostic approach. J Inherit Metab Dis 33:S91-S94.

Dantas MF, Suormala T, Randolph A, Coelho D, Fowler B, Valle D and Baumgartner MR (2005) 3-Methylcrotonyl-CoA carboxylase deficiency: Mutation analysis in 28 probands, 9 symptomatic and 19 detected by newborn screening. Hum Mutat 26:164.

den Dunnen JT and Antonarakis SE (2001) Nomenclature for the description of human sequence variations. Hum Genet 109:121-4.

Fonseca H, Azevedo L, Serrano C, Sousa C, Marcão A and Vilarinho L (2016) 3-Methylcrotonyl-CoA carboxylase deficiency: Mutational spectrum derived from comprehensive newborn screening. Gene 594:203-210.

Forsyth R, Vockley CW, Edick MJ, Cameron CA, Hiner SJ, Berry SA, Vockley J, Arnold GL and Inborn Errors of Metabolism Collaborative (2016) Inborn Errors of Metabolism Collaborative, outcomes of cases with 3-methylcrotonyl-CoA carboxylase (3-MCC) deficiency - report from the Inborn Errors of Metabolism Information System. Mol Genet Metab 118:15-20.

Frisso G, Detta N, Coppola P, Mazzaccara C, Pricolo MR, D'Onofrio A, Limongelli G, Calabrò R, Salvatore F (2016) Functional studies and in silico analyses to evaluate noncoding variants in inherited cardiomyopathies. Int J Mol Sci 17:E1883.

Gallardo ME, Desviat LR, Rodríguez JM, Esparza-Gordillo J, Pérez-Cerdá C, Pérez B, Rodríguez-Pombo $\mathrm{P}$, Criado $\mathrm{O}$, Sanz R, Morton DH et al. (2001) The molecular basis of 3-methylcrotonylglycinuria, a disorder of leucine catabolsim. Am J Hum Genet 68:334-346.

Grünert SC, Stucki M, Morscher RJ, Suormala T, Bürer C, Burda $\mathrm{P}$, Christensen E, Ficicioglu C, Herwig J, Kölker S et al. (2012) 3-methylcrotonyl-CoA carboxylase deficiency. Clinical, biochemical, enzymatic and molecular studies in 88 individuals. Orphanet J Rare Dis 7:31.

Heard GS, Secor McVoy JR and Wolf B (1984) A screening method for biotinidase deficiency in newborns. Clin Chem 30:125-127.

Morscher RJ, Grünert SC, Bürer C, Burda P, Suormala T, Fowler $\mathrm{B}$ and Baumgartner MR (2012) A single mutation in MCCC1 or MCCC2 as a potential cause of positive screening for 3-methylcrotonyl-CoA carboxylase deficiency. Mol Genet Metab 105:602-606.

Rangel-Córdova EA, Martínez-de Villarreal LE and TorresSepulveda R (2009) 3-Methylcrotonyl-CoA carboxylase deficiency detected by tandem mass spectrometry in Mexican population. Medicina Universitaria 11:238-242.

Scolamiero E, Cozzolino C, Albano L, Ansalone A, Caterino M, Corbo G, di Girolamo MG, Di Stefano C, Durante A, Franzese G et al. (2015) Targeted metabolomics in the expanded newborn screening for inborn errors of metabolism. Mol Biosyst 11:1525-1535.

Scolamiero E, Villani GR, Ingenito L, Pecce R, Albano L, Caterino M, di Girolamo MG, Di Stefano C, Franzese I, Gallo G et al. (2014) B12 deficiency detected in expanded newborn screening. Clin Biochem 47:312-317.
Stenson PD, Mort M, Ball EV, Shaw K, Phillips A and Cooper DN (2014) The Human Gene Mutation Database: Building a comprehensive mutation repository for clinical and molecular genetics, diagnostic testing and personalized genomic medicine. Hum Genet 133:1-9.

Sweetman L and Williams JC (2001) Branched chain organic acidurias. In: Scriver CR, Beaudet AL, Sly WS and Valle D (eds) The Metabolic \& Molecular Bases of Inherited Disease. 8th edition. McGraw Hill, New York, pp 2125-2163.

Tanaka K, Hine DG, West-Dull A and Lynn TB (1980a) Gaschromatographic method of analysis for urinary organic acids. I. Retention indices of 155 metabolically important compounds. Clin Chem 26:1839-1846.

Tanaka K, West-Dull A, Hine DG, Lynn TB and Lowe T (1980b) Gaschromatographic method of analysis for urinary organic acids. II. Description of the procedure, and its application to diagnosis of patients with organic acidurias. Clin Chem 26:1847-1853.

Thomsen JA, Lund AM, Olesen JH, Mohr M and Rasmussen J (2015) Is L-carnitine supplementation beneficial in 3methylcrotonyl-CoA carboxylase deficiency? JIMD Rep 21:79-88.

Villani GRD, Gallo G, Scolamiero E, Salvatore F and Ruoppolo M (2016) "Classical organic acidurias": Diagnosis and pathogenesis. Clin Exp Med 17:305-323.

Wajner M, Coelho DM, Ingrassia R, de Oliveira AB, Busanello EN, Raymond K, Flores Pires R, de Souza CF, Giugliani R and Vargas CR (2009) Selective screening for organic acidemias by urine organic acid GC-MS analysis in Brazil: fifteen-year experience. Clin Chim Acta 400:77-81 .

Wajner M, Raymond K, Barschak A, Luft AP, Ferreira G, Domingues G, Chiochetta M, Sirtori L, Goulart L, Pulrolnik V et al. (2002) Detection of organic acidemias in Brazil. Arch Med Res 33:581-585.

Yang L, Yang J, Zhang T, Weng C, Hong F, Tong F, Yang R, Yin $\mathrm{X}, \mathrm{Yu} \mathrm{P}$, Huang X et al. (2015) Identification of eight novel mutations and transcript analysis of two splicing mutations in Chinese newborns with MCC deficiency. Clin Genet 88:484-488

Zanatta Â, Moura AP, Tonin AM, Knebel LA, Grings M, Lobato VA, Ribeiro CA, Dutra-Filho CS, Leipnitz G and Wajner M (2013) Neurochemical evidence that the metabolites accumulating in 3-Methylcrotonyl-CoA carboxylase deficiency induce oxidative damage in cerebral cortex of young rats. Cell Mol Neurobiol 33:137-146.

\section{Internet Resources}

Baumgartner M (2005) 3-Methylcrotonyl-CoA carboxylase deficiency. Orphanet encyclopedia, http://www.orpha.net/data/patho/GB/uk-MCC.pdf.

\section{Supplementary material}

The following online material is available for this article:

Table S1 - Summary of bioinformatics analysis using the Alamut software.

Associate Editor: Angela M. Vianna-Morgante

License information: This is an open-access article distributed under the terms of the Creative Commons Attribution License (type CC-BY), which permits unrestricted use, distribution and reproduction in any medium, provided the original article is properly cited. 